\title{
Conservative Management of Thyroid Eye Disease
}

\author{
Sadaf Humayun Khan ${ }^{1}$, Uzma Malik², Farooq Ahmed ${ }^{3}$, Zahid Kamal Siddiqui', Samavia Munir ${ }^{2}$ and Nawaz \\ Rashid $^{4}$ \\ ${ }^{1}$ Department of Pharmacology, King Edward Medical University, Lahore, Pakistan \\ ${ }^{2}$ Department of Medicine, King Edward Medical University, Lahore, Pakistan \\ ${ }^{3}$ Department of Eye, Mayo Hospital, Lahore, Pakistan \\ ${ }^{4}$ Department of Radiology, Mayo Hospital, Lahore, Pakistan
}

\begin{abstract}
The aim of the study was to determine the result of conservative management of Graves ophthalmopathy in a multi-disciplinary setting by a team of ophthalmologists and endocrinologists. Twenty-seven patients of thyroid eye disease were included in the study who underwent complete ocular examination and complete thyroid biochemical profile. Each patient was advised lubrication with artificial tears, cool compresses, sleeping with elevated head at bed, taping of the eyelids while sleeping and avoidance of smoking. Follow-ups were done at one and six months. EUGOGO classification was used to classify severity of the disease in mild, moderate, severe, and very severe; whereas, clinical activity score (CAS) was implied to categorise the disease activity. Mild to moderate Graves ophthalmopathy responded very well to conservative management. Moderate cases required additional steroids. Whereas, severe and very severe cases require additional surgical intervention.
\end{abstract}

Key Words: Graves ophthalmology, Conservative management, Lubrication, Multidisciplinary approach.

How to cite this article: Khan SH, Malik U, Ahmed F, Siddiqui ZK, Munir S, Rashid N. Conservative Management of Thyroid Eye Disease. J Coll Physicians Surg Pak 2021; 31(05):599-601.

Thyroid eye disease (TED) is an inflammatory autoimmune condition of the orbit having great potential in causing physiological, psychologicaland social consequences. ${ }^{1}$ The thyroid-stimulating hormone (TSH) receptor is the chief target of the immune system, with eye being the most frequent extra thyroidal site of morbidity. ${ }^{2}$ In majority of cases, nearly $80 \%$ hyperthyroidism is present, but it can occur even in the absence of the hyperthyroid state. TED can present before the beginning of thyroid dysfunction, during the course of the disease, or even after the treatment when the patient is euthyroid. ${ }^{3}$ Majority of the patients (90-98\%) have eye lid retraction or lagophthalmos. Ocular irritation, foreign body sensation and photophobia due to dry eyes is also reported. ${ }^{4}$

The management of TED is preferably done in a multidisciplinary setting, by a team of ophthalmologists and endocrinologists. ${ }^{5}$ It was a descriptive study with non-probability purposive sampling, done at Eye Unit-1 and East Medical Ward, Mayo Hospital Lahore from January to December 2018. An Institutional Review Board approval was obtained from King Edward Medical University/Mayo Hospital, Lahore.

Correspondence to: Dr. Sadaf Humayun Khan, Department of Pharmacology, King Edward Medical University, Lahore, Pakistan

E-mail: sadafhumayunkhan@gmail.com

Received: October 09, 2019; Revised: January 04, 2021;

Accepted: February 16, 2021

DOI: https://doi.org/10.29271/jcpsp.2021.05.599
Twenty-seven patients were included in the study after informed consent. Both male and female patients within the age group of 22-60 years were included. Pregnancy and other immune disorders were excluded.

Each patient underwent a complete ocular examination, including visual acuity, slit-lamp examination, applanation tonometry, fundoscopy, color vision, proptosis assessment by Hertel's exophthalmometer and visual field examination. A complete medical checkup and complete thyroid profile (free T ${ }_{3}$, free $\mathrm{T}_{4,}$ and TSH) was also done and anti-thyroid medications were started or adjusted as per their clinical picture. Severity of disease was classified in mild, moderate, severe and very severe using EUGOGO classification; whereas, clinical activity scores (CAS) was implied to categorise the disease activity. All patients were given lubrication with artificial tears, cool compresses, advised sleeping with elevated head at bed, taping of eyelids and avoidance of smoking. Follow-ups were done at 1 and 6 months.

The data was entered into SPSS version 20. Mean and standard deviation was calculated for quantitative variables including age, intraocular pressure, visual acuity right and visual field status. For qualitative variables like gender, corneal status and smoking status, frequencies and percentages were calculated.

Out of total patients, 10 (37.0\%) were males and 17 (63.0\%) females with mean age about $39.26 \pm 10.33$ years. Mean visual acuity of right eye was $4.30 \pm 1.68$ and in the left eye was $4.85 \pm$ 1.77. The visual acuity in right eye of $8(29.6 \%)$ patients was $6 / 6$, 
$7(25.9 \%)$ had $6 / 9$ and $7(25.9 \%)$ had $6 / 12$ whereas, in the left eye the visual acuity of $10(37.0 \%)$ patients was $6 / 9$ and 6 $(22.2 \%)$ had $6 / 6$. The mean intraocular pressure was $21.17 \pm$ $6.20 \mathrm{mmHg}$. Mean intraocular pressure in the right eye was $21.04 \pm 6.58 \mathrm{mmHg}$ and in the left eye was $21.31 \pm 5.93 \mathrm{mmHg}$. Eighteen (66.7\%) patients were non-smokers; whereas, 9 (33.3\%) were smokers. Regarding corneal status, 19 (70.4\%) patients had healthy cornea, $6(22.2 \%)$ had punctate epithelial erosions, 1 (3.7\%) had tectonic graft, and 1 (3.7\%) had slight haziness of cornea. Twenty (74.1\%) patients had normal visual fields; whereas, 7 (25.9\%) had constricted glaucomatous field effects. Fifteen (55.6\%) patients had normal eyelids; whereas, $12(44.4 \%)$ had lid retraction and lid lag.

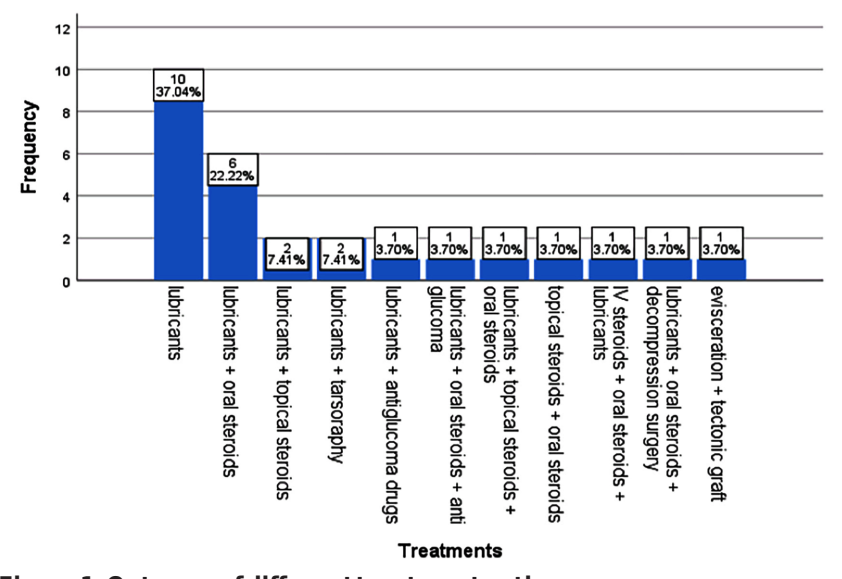

Figure 1: Outcome of different treatment options.

According to EUGOGO classification, 14 (51.9\%) patients were categorised as mild cases, $10(37 \%)$ as moderate cases, 2 $(7.4 \%)$ as severe cases, and $1(3.7 \%)$ patient as very severe case.

At follow-up, mild cases reported having less symptoms and increased rate of patient satisfaction. Moderate cases also required additional steroids. Severe and very severe cases required surgical intervention.

Ten (37\%) patients were treated with only lubricants; whereas, $6(22.2 \%)$ patients were given oral steroids, too. Intravenous steroids and topical anti glaucoma therapy was given to 1 (3.7\%) patient. Orbital decompression surgery was done in 1 (3.7\%) patient. Tarrsoraphy, along with lubricants, was done in $2(7.4 \%)$ patients and 1 (3.7\%) patient underwent evisceration of the eye.

Ophthalmopathy is a fundamental part of Graves disease, having significant psychosocial effects. Patients can end up in having many long term complications. Its treatment depends on how active and severe the course of the disease is in a particular patient. In this study, patients belonged to various age groups and socioeconomic backgrounds, with different degree of ocular involvement. Majority of patients had mild to moderate eye involvement in whom conservative measures are generally helpful.

This study also supports this fact as patients having mild to moderate ocular involvement (88.9\%) were significantly relieved by conservative management. Changes in lifestyle also improved patients' quality of life, reduced the incidence of complications and decreased the need for surgical intervention at an early stage of treatment. Genere and Stan described similar management of mild to moderate cases. ${ }^{5}$ Other foreign researchers including Bartalena et al. also supported this fact. ${ }^{6}$ Systemic review and meta-analysis done by Stiebel-Kalish et al. emphasised on the importance of intravenous steroids in the management of moderate to severe cases. ${ }^{7}$ Severe and very severe cases required additional surgical intervention and were associated by a decrease or loss of vision in one or both the eyes. In addition, surgical treatment was also expensive and required expertise.

The importance of managing patients of TED conservatively cannot be underrated, which benefited the patients, saved the resources and avoided any aggressive treatment at the early stage. The multi disciplinary approach facilitated early diagnosis, prevented complications and improved patient care.

\section{ETHICALAPPROVAL:}

This study was conducted after obtaining approval from the Institutional Ethical Review Committee of King Edward Medical University, Lahore. (No. 22/RC/KEMU dated 15-07-2017).

\section{PATIENTS' CONSENT:}

Informed consents were obtained from all patients.

\section{CONFLICT OF INTEREST:}

Authors declared no conflict of interest.

\section{AUTHORS' CONTRIBUTION:}

SHK: Manuscript writing, data collection, data compilation, literature review, statisticsfinalisation, corresponding author.

UM: Conception of idea, data collection, literature review, final review.

FA: Interpretation of data, literature review, Statistics finalization, final review.

ZKS: Conception of idea, data collection, needful correction and advice, final review.

SM: Data compilation.

NR: Interpretation of data.

\section{REFERENCES}

1. Smith TJ, Hegedüs L. Graves' disease. N Engl J Med 2016; 375(16):1552-65. doi: 10.1056/NEJMra1510030.

2. Bartalena L, Baldeschi L, Boboridis K, Eckstein A, Kahaly GJ, Marcocci C, et al. The 2016 European thyroid association/ European group on Graves' Orbitopathy guidelines for the Management of Graves' Orbitopathy. Eur Thyroid J 2016; 5(1):9-26. doi: 10.1159/000443828.

3. Wiersinga W, Smit T, Van der Gaag R, Koornneef L. Temporal relationship between onset of Graves' ophthalmopathy and onset of thyroidal Graves' disease. J Endocrinol Invest 1988; 11(8):615-9. doi: 10.1007/ BF03350193.

4. Kim YS, Kwak AY, Lee SY, Yoon JS, Jang SY. Meibomian gland dysfunction in Graves' orbitopathy. Can J Ophthalmol 2015; 50(4):278-82. doi: 10.1016/j.jcjo.2015.05.012. 
5. Genere N, Stan MN. Current and emerging treatment strategies for graves' orbitopathy. Drugs 2019; 79(2): 109-24. doi: 10.1007/s40265-018-1045-9.

6. Bartalena L, Marcocci C, Tanda M, Pinchera A. Management of thyroid eye disease. Eur J Nucl Med Mol Imaging 2002;
29(2):S458-S65. doi: 10.1007/s00259-002-0813-6.

7. Stiebel-Kalish H, Robenshtok E, Hasanreisoglu M, Ezrachi D, Shimon I, Leibovici L. Treatment modalities for Graves' ophthalmopathy: Systematic review and metaanalysis. J Clin Endocrinol Metab 2009; 94(8):2708-16. doi: 10.1210/ jc.2009-0376. 